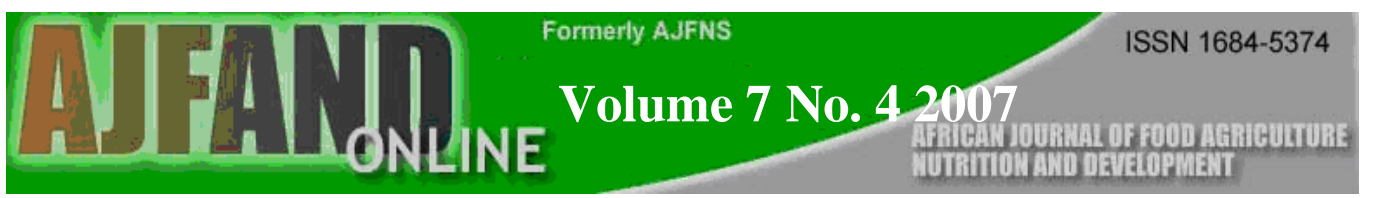

\title{
Pratiques culturales et teneur en éléments anti nutritionnels (nitrates et pesticides) du Solanum macrocarpum au sud du Bénin.
}

By

Assogba-Komlan Francoise. ${ }_{4}$, Anihouvi Prudent. ${ }^{1}$, Achigan Enoch. ${ }^{2}$, Sikirou Rachidatou. ${ }^{3}$, Boko Adrien. ${ }^{4}$, Adje Charlotte. ${ }^{1}$, Ahle Victoire. ${ }^{1}$ Vodouhe Raymond. ${ }^{5}$ Assa Ayémou. ${ }^{6}$

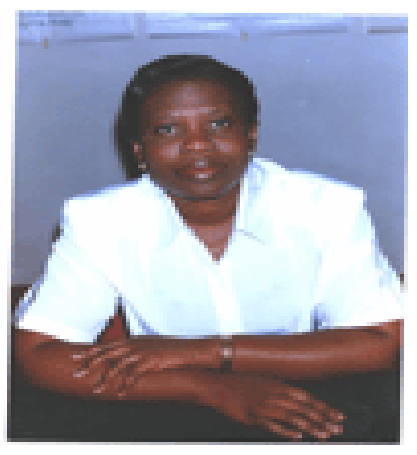

\section{Komlan .F. Assogba*1}

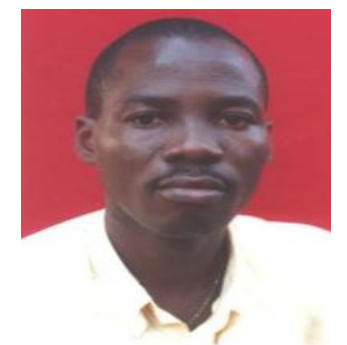

\section{Prudent Anihouvi ${ }^{2}$}

\section{Adresses des correspondants}

ASSOGBA-KOMLAN Françoise, DEA Science du Sol, Doctorat en cours en fertilité et fertilisation des sols, Institut National de Recherches Agricoles du Bénin (INRAB). 01B.P 884 Cotonou, Bénin. Tél : +229 21300264.

E-mail : fakvine60@yahoo.fr

1ANIHOUVI Prudent, Ingénieur Agro-nutritionniste, INRAB. 01B.P 884 Cotonou, Bénin. Tél : +229 21300264.

E-mail : bental2@yahoo.fr

2ACHIGAN Enoch, Ingénieur Agronome, DEA en génétique des plantes, Doctorat en cours sur la diversité génétique des Cucurbitacées. Faculté des Sciences Agronomiques (FSA), Université d'Abomey-Calavi (UAC), Bénin.

E-mail : dachigan@yahoo.fr

3SIKIROU Rachidatou, Ingénieur Agronome, PhD en phytopathologie, Institut National de Recherches Agricoles du Bénin (INRAB), 01B.P 884 Cotonou, Bénin. Tél : +229 20212933.

E-mail : sikirou@bj.refer.org 


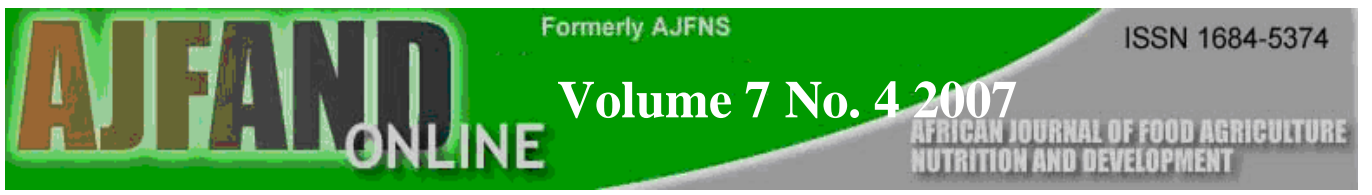

4BOKO Adrien : Ingénieur agrochimiste, Consultant au Laboratoire des Sciences du Sol, Eau et Environnement I Institut National de Recherches Agricoles du Bénin (LSSEE/INRAB). 01B.P 884 Cotonou, Bénin.

Tél. : +229213062 72.

E-mail Syl22ko@yahoo.fr

${ }_{1}$ ADJE Charlotte : Maîtrise Professionnelle en environnement et santé. Tél. +229 95425504 .

E-mail : isaacadje@yahoo.fr

1Victoire AHLE : Technicienne en Production Végétale au Programme de Recherches sur les Cultures

Maraîchères Institut National de Recherches Agricoles du Bénin (INRAB). 01B.P 884 Cotonou, Bénin. Tél. : +229

21300264.

E-mail : manvio20@yahoo.fr

5VODOUHE Raymond Sognon : Regional Coordinator Office for West and Central Africa (WCA) BIOVERSITY, c/o IITA/Benin Research Station, 08 B.P. 0932, Cotonou (Benin). Telephone - Office : 21351991 (BIOVERSITY) Fax: (229) 21354046 (BIOVERSITY)

${ }^{6}$ ASSA Ayémou : Professeur titulaire d'Agro-Pédologie à l'Université de Cocody (Abidjan/ Côte d'ivoire).

Tél. +225224316 21/ +22505077847

E-mail : assaayemou@yahoo.fr 


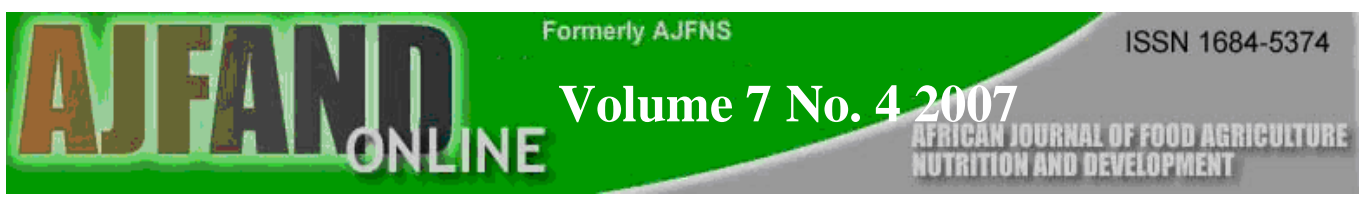

\section{Résumé}

La pression foncière en région urbaine et périurbaine amène beaucoup de producteurs maraîchers à avoir des pratiques culturales qui ne garantissent pas la qualité sanitaire des produits récoltés. Cette étude a analysé les pratiques de fertilisation et de traitements phytosanitaires sur les légumes feuilles traditionnels les plus consommés afin d'identifier ceux à risque. Six (6) échantillons composites de feuilles de S. macrocarpum, répétés deux fois, issus de parcelles ayant reçu des doses de 20,40 et $(20+10+10)$ t/ha de fientes de volaille et de graines de coton, sur un sol ferrallitique faiblement dessaturé au sud du Bénin, ont été analysés au cours des deux premières coupes pour la recherche de nitrates par colorimétrie. Dans la zone de décrue, l'identification et le dosage des pesticides organochlorés par chromatographie en phase gazeuse couplée à un système de détection à capture d'électrons ont été effectués sur dix (10) échantillons de feuilles et un échantillon composite de sol (mélange de trois échantillons de sols) prélevés de façon aléatoire dans les champs maraîchers. Le diagnostic approfondi des pratiques de fertilisation et de traitements phytosanitaires a montré que de fortes doses d'engrais organiques et surtout d'engrais minéraux sont appliquées sur les légumes feuilles dans les zones prospectées. Les taux de nitrate dans les feuilles et dans les sols sont faibles $(<50 \mathrm{mg} / \mathrm{kg}$ de matière fraîche) mais ces taux ont augmenté de la $1^{\text {ère }}$ à la $2^{\text {ème }}$ coupe. Les teneurs en pesticides organochlorés totaux sont comprises entre 0,320 et $2,225 \mu \mathrm{g} / \mathrm{g}$ de légumes secs. En dehors de l'endosulfan et du lindane retrouvés dans les échantillons de légumes, à faibles teneur (respectivement 0,07 et $0,1 \mu \mathrm{g} / \mathrm{g}$ de légumes), les autres pesticides comme le DDT, l'endrine, l'heptachlore, l'aldrine, le dieldrine présentent des teneurs supérieures aux normes Codex appliquées pour le contrôle de la qualité des aliments. Les mêmes pesticides se retrouvent dans les échantillons de sol.

Mots clés : Pratiques culturales, fientes, pesticides organochlorés. 


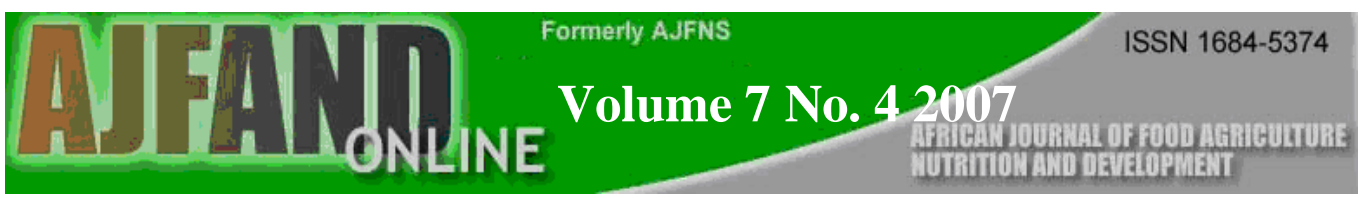

\section{Introduction}

Le Bénin comme beaucoup d'autres pays africains, a connu ces dernières années un développement de l'agriculture urbaine et périurbaine à la suite d'une forte croissance démographique induisant un accroissement des besoins alimentaires. C'est une activité souvent informelle, génératrice de revenus et d'emplois, pratiquée par les couches vulnérables des régions urbaines et périurbaines [1-3]. La production maraîchère et surtout celle des légumes feuilles a pris une part importante dans cette agriculture dans les villes du Sud Bénin [4]. En effet, en matière de cultures maraîchères, les légumes feuilles occupent la deuxième place dans le Sud-Bénin derrière la tomate, avec une superficie de 1.496 ha et une production totale de $10.600 \mathrm{~T}$ [5]. Parmi les cultures maraîchères produites au Bénin, ce sont les légumes feuilles qui sont les plus consommés (62,5\%) et les légumes feuilles traditionnels en y constituent la grande part (89\%). Ces légumes feuilles traditionnels rentrent dans l'alimentation quotidienne de presque tous les Béninois [6].

Le développement de ce type d'agriculture s'est accompagné de plusieurs contraintes dont les plus importantes sont la pression foncière avec pour conséquence la surexploitation des terres et les attaques de ravageurs de toutes sortes occasionnant ainsi d'énormes pertes de productions [7, 8]. Au vu de ces contraintes, il est évident que les conditions de culture de ces légumes feuilles ne soient pas des meilleures: sur-fertilisation organique et/ou minérale, utilisation abusive ou inappropriée de pesticides, etc. Des doses d'engrais organiques (> 40T/ha) et minéraux (>600 kg/ha) sont fréquemment apportées sur Solanum macrocarpum et les ordures ménagères sont également utilisées avec les risques de contamination des sols aux métaux lourds (plomb, cadmium, mercure etc.) [9-12]. De même l'usage des pesticides "coton" comme l'endosulfan, qui sont interdits sur les cultures vivrières et maraîchères, a été signalé sur les légumes $[4,13,14]$. Tous ces facteurs ne garantissent pas la qualité sanitaire des légumes produits. C'est pourquoi la présente étude a été initiée et vise de façon générale à analyser à travers les pratiques culturales et phytosanitaires, la qualité des légumes feuilles traditionnels les plus cultivés et les plus consommés au Bénin. Les objectifs spécifiques sont les suivants :

- Analyser les pratiques de fertilisation et de traitements phytosanitaires en cours sur les légumes feuilles.

- Identifier et évaluer les éléments antinutritionnels (résidus de pesticides et nitrates) présents dans ces légumes feuilles.

\section{Matériel et méthodes}

L'étude a été conduite dans la ville de Cotonou, capitale économique du Bénin et dans six autres villes du sud du Bénin. La région du sud du Bénin bénéficie d'un climat bimodal à deux saisons de pluies et à deux saisons 


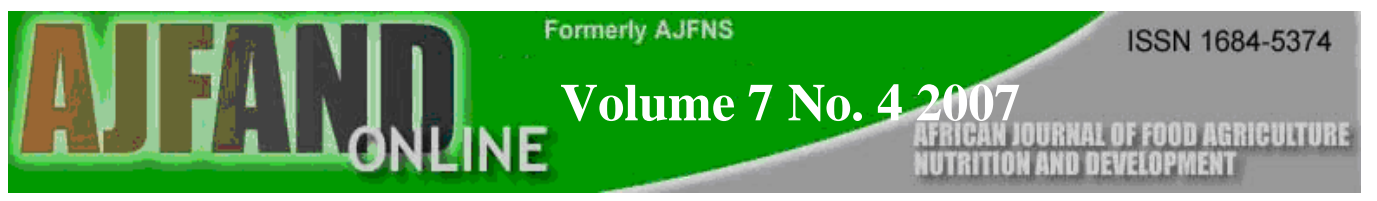

sèches. Les valeurs maximales des températures à Cotonou varient entre 33 et $28^{\circ} \mathrm{C}$. Les valeurs moyennes de l'humidité relative mensuelle varient entre 75 et $90 \%$.

\section{Enquête}

Les zones enquêtées ont été retenues sur la base du niveau de production en légumes feuilles et les recherches documentaires sur les systèmes maraîchers au Sud-Bénin [4, 11, 12, 14, 15]. Ainsi le centre et la périphérie des villes ci-après ont été identifiés pour l'enquête : Cotonou, Ouidah, PortoNovo, Sèmè, Adjohoun (Vallée de l'Ouémé), Grand-Popo et Athiémé.

Un regroupement en zones a été fait non seulement sur la base des caractéristiques écologiques, édaphiques et climatiques mais aussi en tenant compte du zonage effectué par PADAP [14] dans le sud du Bénin qui intègre la pression de l'urbanisation, les surfaces cultivées, la disponibilité en eau et les infrastructures etc. Les localités qui entrent dans les trois grandes zones constituées sont les suivantes:

- Zone du Cordon littoral située aux abords de la mer: Grand-Popo centre, Sèmè

- Zone de décrue: Adjohoun, Athiémé, Grand-Popo rural

- Zone intra et périurbaine: Cotonou, Porto-Novo, Ouidah

L'enquête a été effectuée auprès de 101 producteurs (une trentaine par zone), sélectionnés de façon aléatoire dans les différentes localités ci-dessus citées qui cultivent prioritairement la morelle et/ou l'amarante qui sont des légumes les plus consommés dans le sud du Bénin. Le questionnaire administré a mis l'accent sur les systèmes de culture intégrant les légumes feuilles traditionnels, les pratiques paysannes en matière de fertilisation et de protection phytosanitaire.

\section{Test agronomique}

Le test a été conduit sur la station d'Agonkanmey dans le Sud-Bénin qui est caractérisé par un sol ferrallitique faiblement dessaturé, très sableux en surface (60\% de sable) et un climat de type subéquatorial avec une pluviométrie annuelle moyenne comprise entre 1000 et $1200 \mathrm{~mm}$. La plante test est la morelle (Solanum macrocarpum). Deux (2) types de déchets ont été utilisés : les fientes de poulet et les graines de coton. Les traitements appliqués sont les suivants:

- Apport unique 20T/ha (FP1 et GC1)

- Apport unique 40T/ha (FP2 et GC2)

- Apport fractionné 40T/ha (20-10-10) soit (FP3 et GC3).

Les deux (2) déchets ont été appliqués selon les trois (3) traitements répétés quatre (4) fois. Les parcelles élémentaires ont une superficie de $6 \mathrm{~m}^{2}$. Les 


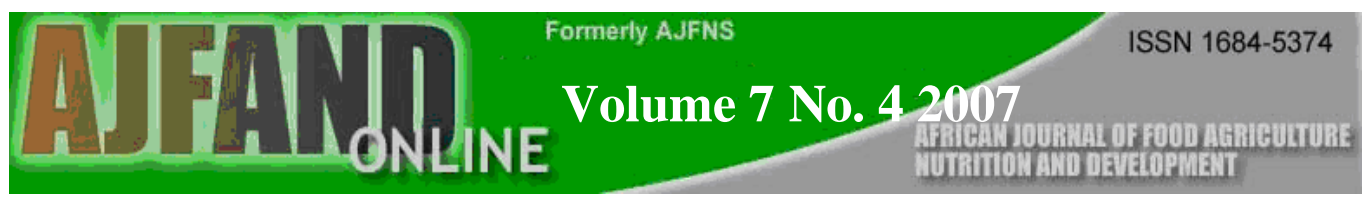

variables mesurées concernent les rendements en feuilles fraîches et les teneurs en nitrates.

\section{Analyses de sols et de feuilles}

Les prélèvements ont été faits à deux niveaux :

- des prélèvements de feuilles issus des tests en milieu contrôlé sur la station d'Agonkanmey pour la recherche de nitrate.

- des prélèvements aléatoires sur les sites maraîchers de sols et de feuilles pour la recherche de nitrate (Cotonou) et de résidus de pesticides (Vallée de l'Ouémé). En effet, ces options ont été faites à cause des pratiques paysannes recensées lors des enquêtes: les apports en fertilisants azotés dépassant les normes à Cotonou et l'utilisation des pesticides tout venant dans la vallée de l'Ouémé.

Pour les analyses de nitrates en station, six (6) échantillons composites de feuilles, répétés deux fois, issus de parcelle ayant reçu des doses de 20, 40 et $(20+10+10) \mathrm{T} / \mathrm{ha}$ de fientes de volaille et de graines de coton, ont été analysés au cours des deux premières coupes. La récolte du S.macrocarpum se fait par coupes successives. Les enquêtes ont révélé que le nombre moyen de coupes est de 3 avec des extrêmes de 12 dans la vallée de l'Ouémé.

Au laboratoire, $5 \mathrm{~g}$ de feuilles lavées à l'eau déminéralisée sont traités après broyage avec de l'acide acétique (2\%). Le filtrat après homogénéisation est conservé au réfrigérateur pour dosage des ions nitrates. Les ions nitrates (NO3 $\left.{ }^{-}\right)$ont été dosés par colorimétrie avec la méthode de salicylate de sodium après extraction dans une solution d'acide acétique à $2 \%$ pour les échantillons de feuilles et dans une solution de $0,1 \mathrm{~N}$ de sulfate de potassium pour les échantillons de sols.

Pour les analyses de résidus de pesticides, dix (10) échantillons de feuilles de S. macrocarpum ont été prélevés sur les plants matures, emballés dans du papier aluminium et conservés au frais dans une glacière jusqu'au laboratoire. Un (1) échantillon composite de sols (mélange de trois échantillons de sols pris au hasard sur un site maraîcher de la vallée de l'Ouémé), a été prélevé à une profondeur de $0-30 \mathrm{~cm}$.

L'extraction des résidus de pesticides a été réalisée à l'extracteur Soxlhet avec le pentane comme solvant. La chromatographie en phase gazeuse couplée à un système de détection à capture d'électrons (CPG/ECD) a été utilisée pour le dosage. 


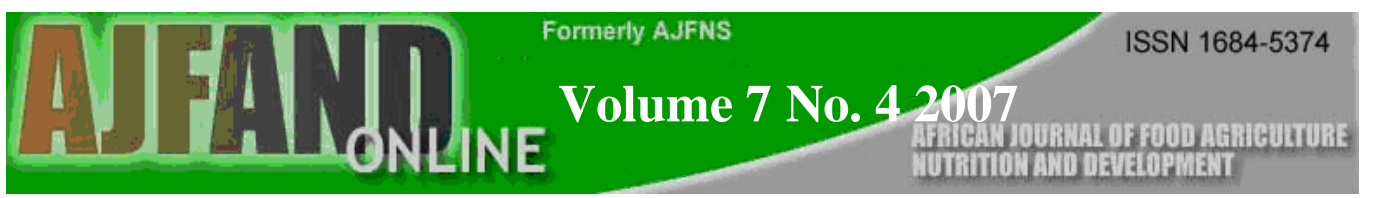

\section{Analyse des données}

Pour les données d'enquête, l'analyse descriptive a été utilisée pour le calcul des moyennes, d'écart type et de pourcentage.

Pour le test agronomique, les facteurs analysés sont : les déchets, les doses et les effets doses (effets directs et en arrière effets). Les effets doses sont mesurés par la première coupe et les arrière-effets qui se décomposent comme suit :

- $\quad$ effet direct + arrière effets pour les apports fractionnés

- arrière effet simple pour les apports uniques.

Ainsi au total, six (6) traitements ont été comparés.

L'analyse de variance a été faite par la méthode ANOVA avec le logiciel SAS version 2000. La PPDS a été utilisée pour comparer les moyennes au seuil de $5 \%$.

\section{RESULTATS}

\section{Résultats d'enquête}

Au cours de l'enquête effectuée dans la région sud du Bénin une analyse des pratiques paysannes de fertilisation et de protection phytosanitaire sur les légumes feuilles a été faite.

\section{Légumes feuilles les plus cultivés}

Solanum macrocarpum (morelle ou "gboma" en langue fon) et Amaranthus hydridus (amarante ou "fotètè") sont les deux légumes feuilles identifiés comme étant les plus cultivés dans la région sud du Bénin. Ces légumes feuilles sont produits par $95 \%$ et $58 \%$ des producteurs enquêtés. Celosia argentea (célosie ou "soma"), Corchorus olitorius (crincrin ou "ninnouwi") et le Vernonia amygdalina (vernonie ou "amavivè") sont produits respectivement par $30 \%, 22 \%$ et $10 \%$ des maraîchers enquêtés.

En considérant l'ensemble des exploitations maraîchères enquêtées au cours de l'année 2003, les superficies moyennes emblavées sont de $643 \mathrm{~m}^{2}$ pour la morelle et de $328 \mathrm{~m}^{2}$ pour l'amarante. Les résultats d'enquête ont en outre montré qu'environ $60 \%$ des producteurs consacrent plus de $50 \%$ de leurs superficies à la production de ces deux légumes feuilles alors que $17 \%$ des producteurs leur en réservent moins de $25 \%$. Tout ceci démontre l'importance que revêtent ces deux légumes feuilles dans les systèmes de cultures maraîchères du Sud-Bénin. 


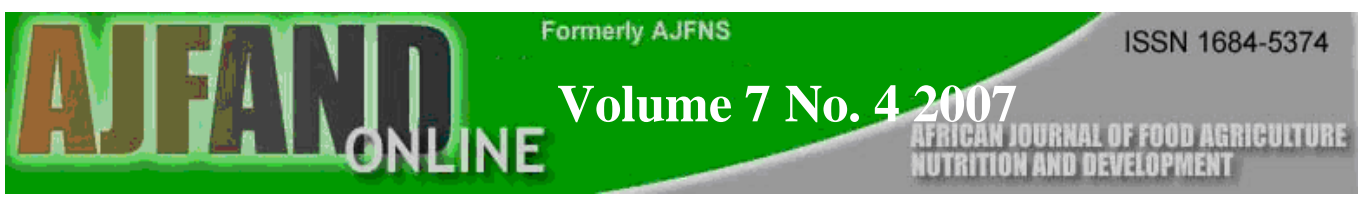

\section{Pratiques culturales sur les légumes feuilles}

\section{Fertilisation organique et ou minérale}

Le recours à la fertilisation organique et minérale est systématique dans les zones intra urbaines, périurbaines et du Cordon littoral alors qu'il demeure très marginal dans les zones de décrue. En effet, 16 et $17 \%$ des producteurs de la zone de décrue utilisent respectivement les engrais minéraux et organiques.

Trois types d'engrais organiques sont identifiés comme étant les principales sources de matière organique utilisées par les maraîchers du Sud-Bénin dans la production des légumes feuilles: fientes de poulet $(71,4 \%$ des producteurs enquêtés), bouse de vache $(17,5 \%)$ et ordures ménagères $(11,1 \%)$. L'application directe sans compostage de ces différents types d'engrais organiques est la pratique la plus observée.

Les doses et fréquences d'apport des engrais organiques et minéraux sont présentées dans le tableau 1. Ces valeurs ne concernent que les fientes de poulet et la bouse de vache. Les ordures ménagères n'ont pas été prises en compte du fait de la grande difficulté au cours de l'étude à estimer les quantités utilisées. En effet, les quantités apportées dépendent de la main d'œuvre utilisée (âge et sexe), le lieu d'entreposage, les bols et bassines utilisés etc. L'analyse de ce tableau montre que la morelle bénéficie de plus d'amendement organique que l'amarante. L'engrais organique est apporté en moyenne une fois à l'amarante et 1,5 fois à la morelle. Par ailleurs, les doses d'engrais organiques les plus élevées sont enregistrées en zones du Cordon littoral (28,85 t/ha) et de l'intra et périurbain (26,47 t/ha). Les doses d'engrais organiques appliquées sont presque partout supérieures à celle recommandée par la recherche (20 t/ha) quel que soit le type de légume feuille.

L'urée est l'engrais minéral le plus utilisé sur les légumes feuilles.79\% des producteurs l'utilise seul et $21 \%$ l'utilise en mélange avec le NPK surtout pour la production de la morelle.

L'urée est apportée de façon fractionnée en moyenne deux fois pendant tout le cycle de culture quelle que soit la zone.

La morelle bénéficie plus d'apport d'urée que l'amarante. En effet, les doses d'urée apportées à la morelle font en moyenne deux fois celles appliquées à l'amarante quelle que soit la zone. Les doses d'application d'urée les plus élevées sont observées sur le Cordon littoral $(1150 \mathrm{~kg} / \mathrm{ha})$ et les plus faibles dans la zone de décrue $(360 \mathrm{~kg} / \mathrm{ha})$. Quelle que soit la zone, les doses d'urée sont supérieures à celle recommandée par la recherche nationale sur les légumes feuilles (75 - $150 \mathrm{~kg} / \mathrm{ha})$ [27]. Les enquêtes ont révélé des applications dépassant 5 fois la dose recommandée. 
La distribution des producteurs suivant les doses d'urée apportées est présentée au tableau 3. Ce tableau que près de $83 \%$ des producteurs de morelle et $50 \%$ des producteurs d'amarante apportent des doses d'urée six fois supérieures à la dose recommandée. Les doses les plus appliquées sont comprises entre 300 et $600 \mathrm{~kg} / \mathrm{ha}$ pour la morelle (34\% des producteurs de morelle) et 150 et $600 \mathrm{~kg} / \mathrm{ha}$ pour l'amarante $(70 \%$ des producteurs d'amarante). Des doses dix fois supérieures à la dose recommandée sont enregistrées chez environs $34 \%$ des producteurs de morelle et $6 \%$ des producteurs d'amarante.

\section{Pratiques phytosanitaires sur les légumes}

Les problèmes phytosanitaires constituent l'une des contraintes qui limitent la production de légumes feuilles dans le Sud-Bénin. Tous les maraîchers enquêtés ont signalé des attaques de ravageurs (acariens, chenilles) et maladies (pourriture dues au sclerotium, nématodes) sur les légumes feuilles. La lutte chimique est la principale méthode utilisée par les maraîchers quelle que soit la zone.

Les pratiques en matière d'utilisation de pesticides sont des plus dangereuses. En effet, les maraîchers pulvérisent le plus souvent les légumes avec une diversité d'insecticides et de fongicides à des doses inappropriées. La liste des produits de synthèse les plus utilisés par zone, le pourcentage de producteurs utilisant chaque type de produit ainsi que les doses et fréquences moyennes d'applications sont présentés dans le tableau 4. Dans la zone de décrue, les insecticides coton comme Dursban (chlorpyriphos-éthyl), Cotalm (cyperméthrine + diméthoate), endosulfan etc., interdits sur les légumes sont beaucoup utilisés par un nombre élevé de producteurs

Les doses appliquées varient d'un producteur à l'autre. La majorité des producteurs appliquent différentes doses jusqu'à obtenir la dose efficace. Le nombre d'applications est variable selon les expériences des producteurs. Pour certains, le traitement se fait à chaque fois qu'il y a apparition d'insectes sur la plante ; pour d'autres il faut traiter tous les trois jours sans attendre l'apparition des insectes.

Les doses de pesticides appliquées par traitement sont en général supérieures à celles recommandées quelle que soit la zone ou quel que soit le pesticide (tableau 4). Les doses les plus élevées sont enregistrées au niveau des zones intra et périurbaines et littorales. Les doses moyennes appliquées dans lesdites zones sont comprises entre 1,5 et 5 fois celles recommandées. Les doses notées au niveau des zones de décrue sont au contraire proches de celles qui sont recommandées pour les quelques produits autorisés (TopsinM, orthène etc.). Cependant les fréquences d'applications les plus élevées sont notées dans la zone de décrue (5 fois en moyenne) avant la première coupe soit en deux mois et demi. 


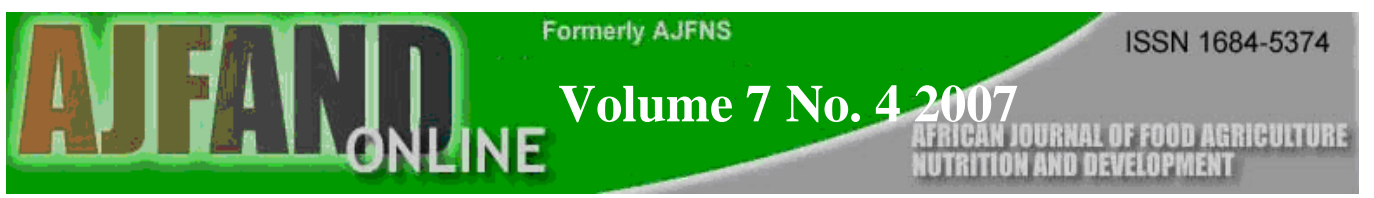

\section{Résultats du test agronomique}

Sources de matière organique et rendements en feuilles de morelle.

Les analyses statistiques ont montré qu'au seuil de 5 p.c., les interactions entre doses et déchets ne sont pas significatives c'est-à-dire que les deux facteurs considérés sont indépendants. C'est pourquoi le tableau 5 présente les effets (doses et déchets).

Les résultats obtenus entre la première coupe : effet et la deuxième coupe : arrière effet sont significativement différents au seuil de 5 p.c. quel que soit le paramètre mesuré, toutes doses et tous déchets confondus. Les constats sont que :

- Les premières applications de déchets contribuent à l'élargissement des feuilles

- Les arrières effets permettent une augmentation du nombre des feuilles mais celles-ci sont plus petites

- Les arrières effets donnent les rendements les plus élevés.

\section{Dose d'engrais organique et teneurs en nitrates}

La figure 1 présente l'effet des sources de matière organique et des doses sur la teneur en nitrates des feuilles de morelle. La comparaison des teneurs en nitrates des feuilles du point de vue statistique, montre qu'il n'y pas eu de différence significative entre les traitements aussi bien à la première qu'à la deuxième coupe. Les doses et mode d'apport de fientes n'ont pas influencé la teneur en nitrates des feuilles de Solanum macrocarpum" pour les deux coupes effectuées. 


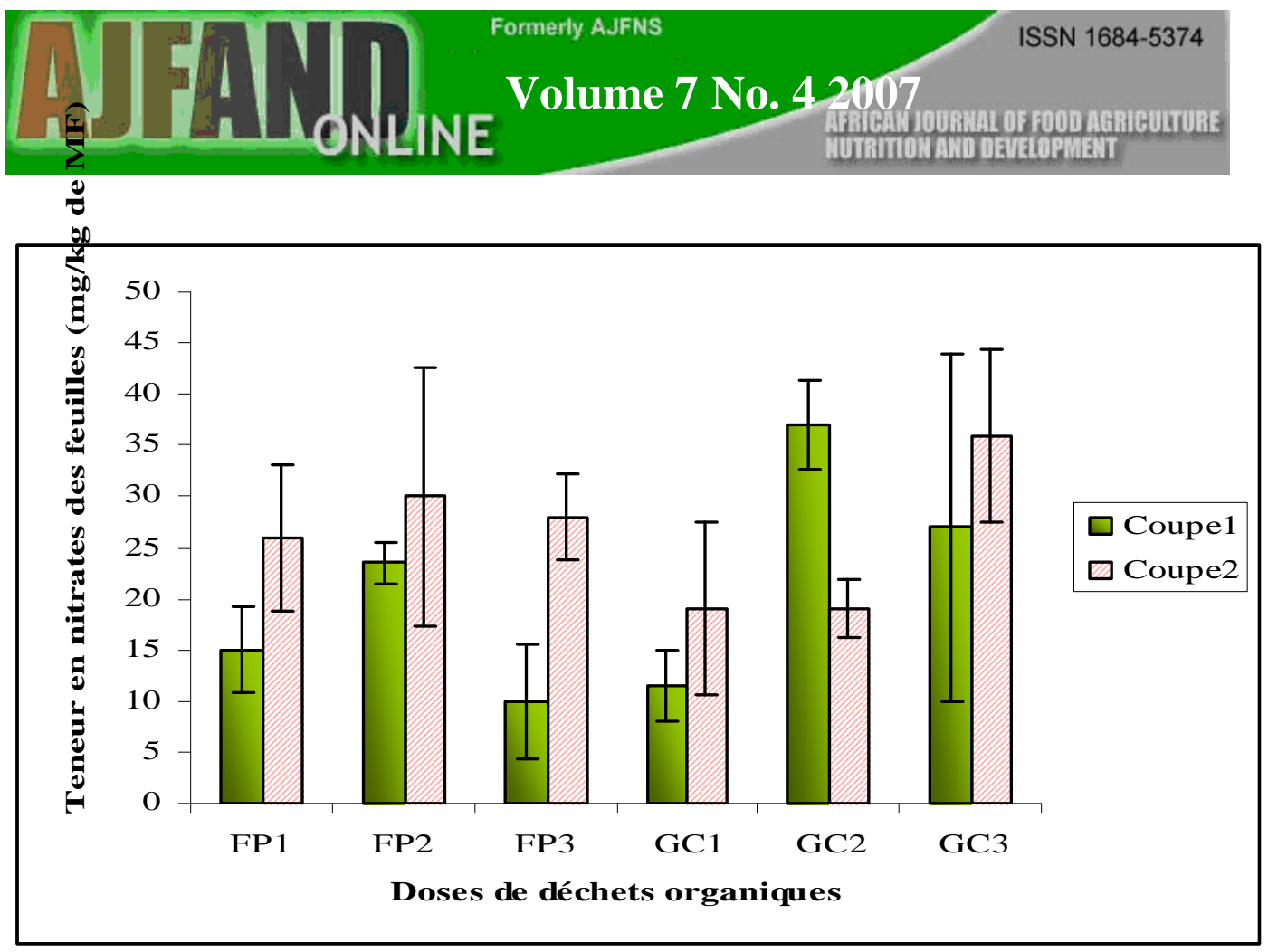

Figure 1: Influence des apports organiques sur la teneur en nitrate des feuilles en milieu contrôlé

FP1 $=20 \mathrm{~T} /$ ha de fientes de poulet en apport unique $\mathrm{FP} 2=40 \mathrm{~T} /$ ha de fientes de poulet en apport unique FP3 $=40$ soit $(20+10+10)$ T/ha en apport fractionné $\mathrm{GC1}=20 \mathrm{~T} / \mathrm{ha}$ de graines de coton en apport unique $\mathrm{GC2}=40 \mathrm{~T} / \mathrm{ha}$ de graines de coton en apport unique GC3 $=40$ soit $(20+10+10)$ T/ha de graines de coton en apport fractionné

Les teneurs en nitrate même si elles sont faibles, ont augmenté lorsqu'on passe de 20 à $40 \mathrm{~T} / \mathrm{ha}$. L'apport fractionné de 40T/ha a donné des teneurs statistiquement égales à l'apport unique de $40 \mathrm{~T} / \mathrm{ha}$ mais avec des tendances à la baisse beaucoup plus prononcées pour les fientes que pour les graines de coton.

A l'instar des rendements, la concentration en nitrates des feuilles d'une manière générale a augmenté quels que soient les traitements de la première à la deuxième coupe surtout dans le traitement pour les fientes de poulets.

\section{Doses de résidus de pesticides dans les feuilles et le sol}

\section{Nitrates}

Les échantillons de sol prélevés dans la Vallée de l'Ouémé présentent des concentrations en nitrates plus élevées que ceux prélevés à Cotonou (respectivement 16,16 et $3,91 \mathrm{NO}_{3}^{-} \mathrm{mg} / \mathrm{kg}$ ). Les échantillons de feuilles 


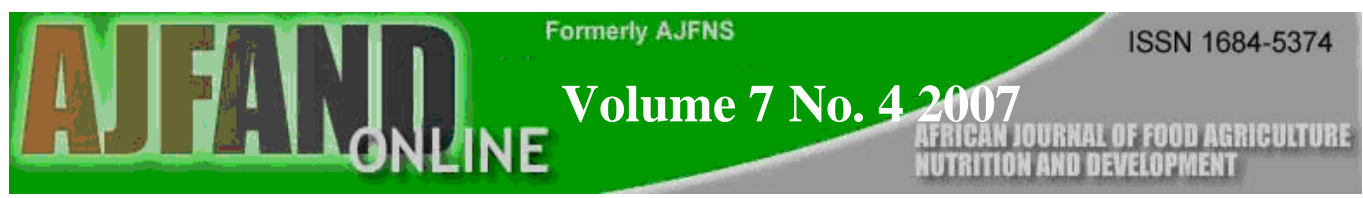

prélevés présentent des teneurs en nitrates variables. La teneur moyenne est de 13,92 mg/kg de feuilles fraîches dans la Vallée de l'Ouémé et de 16,89 $\mathrm{mg} / \mathrm{kg}$ à Cotonou. Tous les échantillons de feuilles ont accumulé des nitrates, mais à des taux inférieurs aux normes admises par l'OMS/FAO (500 mg/kg) [28].

\section{Résidus de pesticides}

Les analyses de feuilles et de sols révèlent une contamination par les pesticides organochlorés comme nous le montrent les tableaux 6 et 7 .

Les pesticides organochlorés détectés dans les légumes sont le DDT, l'Endrine, l'Heptachlore, l'Aldrine, l'Endosulfan, le Dieldrine et le Lindane. Les mêmes pesticides se retrouvent dans l'échantillon composite de sol.

Le DDT, l'endosulfan et l'heptachlore ont été détectés dans tous les échantillons de légumes. En dehors de l'endosulfan et du lindane retrouvés dans les échantillons de légumes, à faibles teneurs, les autres pesticides présentent des teneurs supérieures aux normes Codex appliquées pour le contrôle de la qualité des aliments.

\section{DISCUSSION}

Les résultats ont montré que les pratiques de fertilisation varient suivant les zones. Alors que l'apport d'engrais organique et minéraux est systématique dans les zones du Cordon littoral et de l'intra et périurbain, il est peu fréquent en zones de décrue. En effet, avec la crue, beaucoup d'éléments fertilisants sont transportés et déposés au niveau des sols. Après le retrait de l'eau, les sols des zones de décrue (sols alluvionnaires) sont pourvus par une richesse en éléments fertilisants. Cette fertilisation naturelle justifie la faible proportion de producteurs s'adonnant à la pratique de fertilisation.

Dans la zone intra et périurbaine où les ordures ménagères sont encore utilisées, le triage de ces ordures, souvent mal exécuté, augmente les risques de pollution des espaces cultivés par les matières non biodégradables et de contamination des légumes par les métaux lourds [16].

Quelle que soit la zone, la morelle bénéficie de plus d'apports organique et minéral que l'amarante. Ceci s'explique par le fait que le nombre de coupes pratiquées sur la morelle est supérieur à celui de l'amarante.

Les doses de fertilisation les plus élevées sont observées dans les zones du Cordon littoral et de l'intra et périurbain. En effet, les sols très sableux de ces zones sont pauvres en matière organique. De même, les besoins d'intensification à cause de la pression foncière, exigent des apports élevés en intrants pour une augmentation de la productivité. Enfin, ces types de sols favorisent une lixiviation rapide des éléments nutritifs vers les horizons inférieurs. 
Ces pratiques de surdosage observées surtout au niveau des zones de l'intra, périurbain et du Cordon littoral mettent en évidence les risques de surfertilisation azotée et d'accumulation de nitrates dans les feuilles de morelle et d'amarante. En effet, la sur-fertilisation azotée peut conduire à une accumulation de nitrates dans les feuilles, surtout celles utilisées comme épinard et laitue $[18,19]$. Ce qui ne saurait rester sans conséquences sur la santé des consommateurs. En effet, une partie des nitrates ingérés peut être transformée en nitrites responsables des troubles respiratoires surtout chez les jeunes enfants avec pour conséquence la méthémoglobinémie [17, 20]. Des études ont prouvé de façon irréfutable que les nitrates se combinent aux amines pour former des composés cancérogènes appelés nitrosamines [21]. Cependant les résultats d'analyse de nitrates au niveau des feuilles ont révélé des taux très faibles comparés aux normes admises par l'OMS (500 mg/kg). Certains auteurs ont déjà fait état de faibles taux de nitrates au niveau des légumes produits à Cotonou [22, 23].

Les légumes produits dans le Sud-Bénin présentent donc très peu de risques d'intoxication en nitrates. Néanmoins les risques de pollution de la nappe phréatique par les nitrates sont probables à cause des sols très filtrants observés dans le sud du Bénin.

Les résultats d'enquête ont montré que les risques de contamination des légumes par les pesticides sont plus grands dans les zones de décrue. En effet, en se référant à l'Index Phytosanitaire, il ressort que la plupart des pesticides utilisés en zones de décrue (Cotalm, Dursban) notamment dans la vallée de l'Ouémé sont des insecticides « coton » à forte toxicité, prohibés sur les cultures vivrières [24]. De même, les fréquences de traitement les plus élevées sont notées au niveau de cette zone. Par contre les pesticides les plus utilisés en zones littorales (Topsin-M, Orthène, Banko Plus) et en zones intra et périurbaines (Manèbe, Decis) font partie de ceux recommandés en maraîchage.

Les paramètres mesurés en plein champ : la largeur des feuilles et le nombre de feuilles, les rendements en matière sèche et fraîche, ont évolué différemment selon qu'on considère les effets directs ou les arrières effets. Les feuilles les plus larges sont obtenues à la première coupe quel que soit le type de déchets. Ce qui suppose que l'azote contenu dans les déchets a été surtout utilisé pour un bon développement des feuilles. En effet, l'azote est un facteur essentiel de croissance des plantes surtout au niveau des feuilles et des tiges [25]. Après la première coupe, le nombre de ramifications augmente avec aussi une augmentation du nombre de feuilles. Malheureusement toutes ces feuilles ne peuvent pas bénéficier d'une bonne photosynthèse; leur développement est donc limité. Le nombre de feuilles ayant augmenté, le rendement augmente également d'où le résultat que l'arrière effet donne des rendements supérieurs à l'effet direct quels que soient les doses et les types de déchets. Le rendement élevé obtenu au niveau de l'arrière effet de la dose 


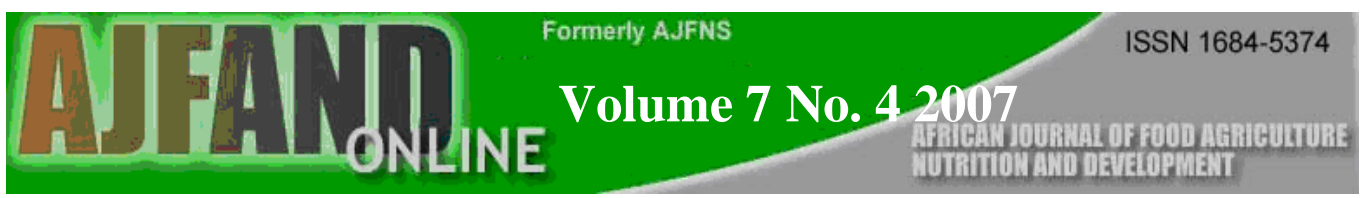

fractionnée de 40 T/ha peut être expliqué par la mise à disposition progressive des éléments nutritifs à des moments où la plante en a besoin, la reconstitution du stock organique par l'apport répété de déchets au fur et à mesure que les eaux d'irrigation contribuent au lessivage des éléments nutritifs et le mulch permanent occasionné par les apports successifs de déchets organiques. Ce mulch maintient l'humidité du sol et la diminution du lessivage au niveau de ces parcelles [26].

Les doses et mode d'apport de déchets n'ont pas influencé la teneur en nitrates des feuilles de Solanum macrocarpum. En examinant les taux de nitrates dans les feuilles, on peut déduire que les plants suivant les doses de déchets apportées, n'utilisent pas de la même manière les nitrates prélevés dans le sol. D'autres facteurs incontrôlables peuvent être à l'origine de cette variabilité comme le démontre les écarts enregistrés : ensoleillement, développement de la plante, état du sol, etc.

Comme les rendements, la concentration en nitrates des feuilles d'une manière générale a augmenté de la première à la deuxième coupe. Cela peut être dû à la décomposition des déchets organiques, qui libère progressivement l'azote minéral prélevé par la plante.

Les fortes doses (40T/ha) n'ont pas induit des teneurs élevées en nitrates des feuilles par rapport aux faibles doses. Ceci laisse supposer que la décomposition des déchets n'est pas encore totale compte tenu de la quantité apportée. Ainsi, la plante utilise la majeure partie de l'azote minéral absorbé pour son développement. Les rendements ont donc augmenté de la première à la $2^{\text {ème }}$ coupe. Cependant l'accumulation des nitrates dans les feuilles des parcelles ayant reçu 40T/ha, pourrait être accentuée au moment où les plants auraient atteint un stade de développement maximal et que la décomposition serait avancée. En effet, lorsque les rendements finissent par atteindre un seuil malgré l'apport d'azote, la teneur en nitrates des légumes continue d'augmenter. A ce stade, les végétaux au lieu d'utiliser les nitrates à des fins structurales ou les incorporer à la chlorophylle et à d'autres composés, ils les gardent en réserve dans leurs tissus foliaires sous forme de nitrates [19, 21].

Les résultats d'analyses de résidus de pesticides des échantillons de sols et feuilles prélevés dans la vallée de l'Ouémé montrent l'existence des risques de contamination par les produits phytosanitaires. Cependant ces risques existent également dans les zones du Cordon littoral et de l'intra et périurbain à cause du surdosage observé même si les produits utilisés sont recommandés en maraîchage. D'autres études ont déjà fait mention d'une utilisation inappropriée ou abusive de pesticides en maraîchage dans le SudBénin $[4,13,14]$. Le DDT, l'endosulfan et l'heptachlore ayant été détectés dans tous les échantillons de légumes, on pourrait conclure une utilisation généralisée de ces trois types de pesticides au niveau de tous les sites. Ces trois pesticides pourraient constituer de bons indicateurs de suivi de pollution par les pesticides organochlorés dans la vallée de l'Ouémé. 


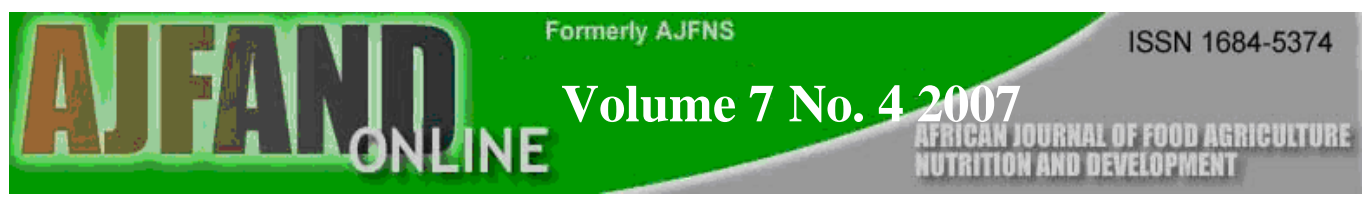

En dehors de l'endosulfan et de la lindane retrouvés dans les échantillons de légumes, à faibles teneurs, les autres pesticides présentent des teneurs supérieures aux normes Codex appliquées pour le contrôle de la qualité des aliments. Les analyses étant effectuées sur des échantillons de légumes à l'état brut; les risques d'intoxication des consommateurs par les produits de maraîchage de la vallée de l'Ouémé sont réels.

\section{CONCLUSION}

La morelle (Solanum macrocarpum) et l'amarante (Amaranthus cruentus) sont les deux principaux légumes feuilles traditionnels les plus cultivés au Sud-Bénin. Cette étude a permis de montrer que les pratiques actuelles sur ces légumes feuilles participent non seulement à détériorer leur qualité nutritionnelle mais aussi à la dégradation de l'environnement. En effet, des pratiques de sur-fertilisation (organique et/ou minérale) et d'utilisation abusive ou inappropriée de pesticides sur ces légumes feuilles notées lors des enquêtes, ne sont pas de nature à garantir la qualité nutritionnelle de la morelle et de l'amarante.

Pour la production de feuilles dans les sols sableux du sud du Bénin, les doses de 20 et $40 \mathrm{~T} / \mathrm{ha}$ et la fréquence d'apport des fientes ou des graines de coton ont peu influencé les rendements et les teneurs en nitrates des feuilles. Bien que des études récentes aient montré que la consommation des légumes riches en nitrates pourrait améliorer certaines réactions immunitaires de l'organisme à des agents pathogènes [17], il est important de tenir compte de l'augmentation de ces taux dans les feuilles consommées dans les coupes successives, pratiques souvent observées dans la gestion des légumes feuilles.

Les concentrations en pesticides organochlorés dans les feuilles sont supérieures aux normes Codex appliquées pour le contrôle de la qualité des aliments. Les risques d'intoxication des consommateurs par les pesticides sont grands et réels. Une action conjuguée des différents acteurs en agriculture urbaine est indispensable pour une sécurité alimentaire durable au sud du Bénin.

NOS REMERCIEMENTS VONT A :

- I'IPGRI bureau régional de l'Afrique de l'Ouest qui a permis la réalisation de cette étude à travers un appui financier,

- tous les chercheurs et techniciens de I'INRAB qui ont contribué à un moment donné à cette étude,

- aux autorités de I'INRAB qui ont soutenu cette étude. 


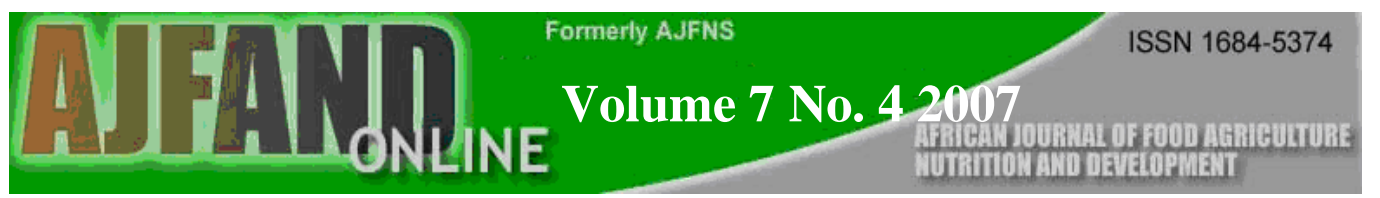

Tableau 1: Dose et fréquence moyennes d'apport de l'engrais organique par zone sur la morelle et l'amarante

\begin{tabular}{|c|c|c|c|c|}
\hline \multirow[t]{2}{*}{ Zones } & \multicolumn{2}{|l|}{ Morelle } & \multicolumn{2}{|l|}{ Amarante } \\
\hline & Dose (t/ha) & $\begin{array}{l}\text { Fréquence } \\
\text { d'apport }\end{array}$ & Dose (t/ha) & $\begin{array}{l}\text { Fréquence } \\
\text { d'apport }\end{array}$ \\
\hline Cordon littoral & 28,8 (13) & 1,1 & $22,4(4)$ & 1,0 \\
\hline $\begin{array}{l}\text { Intra et } \\
\text { périurbain }\end{array}$ & 26,4 (34) & 1,4 & $15,9(33)$ & 1,1 \\
\hline Décrue & $23,3(8)$ & 1,4 & $23,3(6)$ & 1,3 \\
\hline Sud-Bénin & $26,6(55)$ & 1,3 & $17,5(43)$ & 1,1 \\
\hline
\end{tabular}

$\underline{\text { Tableau 2 }}$ : Dose et fréquence moyennes d'apport de l'urée par zone

\begin{tabular}{|c|c|c|c|c|}
\hline \multirow[t]{2}{*}{ Zones } & \multicolumn{2}{|l|}{ Morelle } & \multicolumn{2}{|l|}{ Amarante } \\
\hline & Dose (t/ha) & $\begin{array}{l}\text { Fréquence } \\
\text { d'apport }\end{array}$ & Dose (t/ha) & $\begin{array}{l}\text { Fréquence } \\
\text { d'apport }\end{array}$ \\
\hline Cordon littoral & $\mathbf{1 1 5 1 , 5}(15)$ & 2,1 & $420,8(4)$ & 1,0 \\
\hline $\begin{array}{l}\text { Intra et } \\
\text { périurbain }\end{array}$ & 636,6 (39) & 2,4 & $361,1(39)$ & 1,4 \\
\hline Décrue & $361,9(7)$ & 2,1 & $206,7(5)$ & 1,2 \\
\hline Sud-Bénin & $439,0(61)$ & 2,3 & $210,0(48)$ & 1,3 \\
\hline
\end{tabular}

(): Effectif des producteurs utilisant l'urée par zone

Tableau 3 : Distribution des producteurs suivant les doses d'urée apportées

\begin{tabular}{lllll}
\hline $\begin{array}{l}\text { Dose d'urée } \\
\text { (kg/ha) }\end{array}$ & Morelle & \multicolumn{3}{c}{ Amarante } \\
\cline { 2 - 5 } & Effectif & $\begin{array}{l}\text { Pourcentage } \\
(\%)\end{array}$ & Effectif & $\begin{array}{l}\text { Pourcentage } \\
(\%)\end{array}$ \\
$75-150$ & 3 & 4,92 & 7 & 14,58 \\
& & & & \\
$150-300$ & 7 & 11,47 & $\mathbf{1 7}$ & $\mathbf{3 5 , 4 2}$ \\
$300-600$ & $\mathbf{2 1}$ & $\mathbf{3 4 , 4 3}$ & $\mathbf{1 7}$ & $\mathbf{3 5 , 4 2}$ \\
$600-750$ & 9 & 14,75 & 4 & 8,33 \\
$750-1125$ & $\mathbf{1 3}$ & $\mathbf{2 1 , 3 1}$ & 3 & 6,25 \\
$>1125$ & 8 & 13,12 & 0 & 0 \\
Total & 61 & 100 & 48 & 100 \\
\hline
\end{tabular}




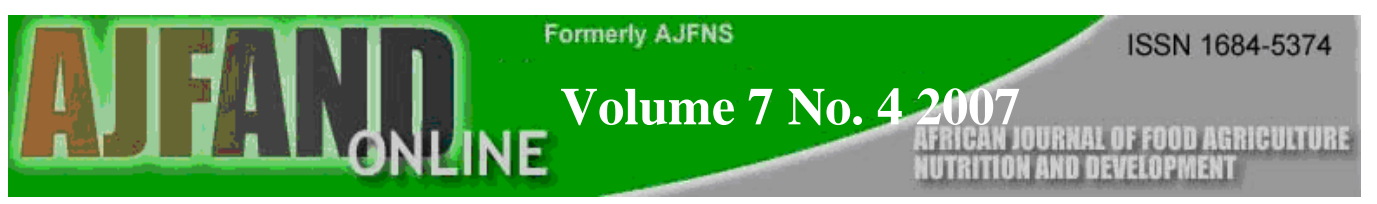

Tableau 4 : Doses et fréquences moyennes d'applications des pesticides les plus utilisés par zone

\begin{tabular}{|c|c|c|c|c|}
\hline Zones & $\begin{array}{l}\text { Produits } \\
\text { phytosanitaires }\end{array}$ & $\begin{array}{l}\text { Dose } \\
\text { moyenne } \\
\text { (g ou l/ha) }\end{array}$ & $\begin{array}{l}\text { Fréquence } \\
\text { d'application }\end{array}$ & $\begin{array}{l}\text { Dose } \\
\text { recommandée }\end{array}$ \\
\hline \multirow{3}{*}{ Cordon littoral } & Topsin-M & $\begin{array}{l}\mathbf{2 1 8 6 , 0} \mathbf{g} / \mathrm{ha} \\
(416-4956)\end{array}$ & $3,5(3-4)$ & $1400,0 \mathrm{~g} / \mathrm{ha}$ \\
\hline & Orthène & $\begin{array}{l}1,025 \mathrm{l} / \mathrm{ha} \\
(0,05-2)\end{array}$ & $4,0(3-5)$ & $0,8 \mathrm{l} / \mathrm{ha}$ \\
\hline & Banko plus & $\begin{array}{l}\mathbf{1 8 5 5 , 0} \mathbf{g} / \mathbf{h a} \\
(1250-2460)\end{array}$ & $3,5(2-5)$ & $500,0 \mathrm{~g} / \mathrm{ha}$ \\
\hline \multirow{3}{*}{ Décrue } & Cotalm & $\begin{array}{l}1,3 \mathrm{l} / \mathrm{ha} \\
(0,05-3)\end{array}$ & $5,5(3-8)$ & $1,0 \mathrm{l} / \mathrm{ha}$ \\
\hline & Dursban & $\begin{array}{l}1,2 \mathrm{l} / \mathrm{ha} \\
(0,06-3)\end{array}$ & $5,0(3-7)$ & $1,0 \mathrm{l} / \mathrm{ha}$ \\
\hline & Orthène & $\begin{array}{l}1,0 \mathrm{l} / \mathrm{ha} \\
(0.05-2)\end{array}$ & $4,0(3-5)$ & $0,8 \mathrm{l} / \mathrm{ha}$ \\
\hline \multirow{2}{*}{$\begin{array}{l}\text { Intra } \\
\text { périurbain }\end{array}$} & & $\begin{array}{l}\text { 2041,9 g/ha } \\
(208-8680)\end{array}$ & $3(2-4)$ & $1600,0 \mathrm{~g} / \mathrm{ha}$ \\
\hline & Decis & $\begin{array}{l}4,9 \text { l/ha } \\
(0,125-20,8)\end{array}$ & $3(1-5)$ & $1,0 \mathrm{l} / \mathrm{ha}$ \\
\hline
\end{tabular}

( ) : Min-Max

Tableau 5: Effet direct et arrière effet des facteurs déchets et doses sur la croissance des plants de morelle.

\begin{tabular}{|c|c|c|c|c|}
\hline & \multicolumn{4}{|c|}{ Paramètres mesurés } \\
\hline Effets & $\begin{array}{l}\text { Hauteur plant } \\
(\mathrm{cm})\end{array}$ & $\begin{array}{l}\text { Largeur } \\
\text { feuille }(\mathrm{cm})\end{array}$ & $\begin{array}{l}\text { Nombre de } \\
\text { feuilles }\end{array}$ & $\begin{array}{l}\text { Rendements } \\
\text { Feuilles fraîches }\end{array}$ \\
\hline Effet direct & $26.99 \pm 4.63 \mathrm{~b}$ & $\begin{array}{l}15.88 \pm 1.48 \\
a\end{array}$ & $\begin{array}{l}33.44 \pm 5.36 \\
b\end{array}$ & $14.68 \pm 5.99$ b \\
\hline Arrière effet & $33.17 \pm 4.12 \mathrm{a}$ & $\begin{array}{l}11.92 \pm 1.72 \\
b\end{array}$ & $\begin{array}{l}47.20 \pm 8.62 \\
a\end{array}$ & $20.32 \pm 4.03 \mathrm{a}$ \\
\hline
\end{tabular}

Tableau 6: Teneurs en pesticides organochlorés (en $\mu \mathrm{g} / \mathrm{kg}$ ) dans les échantillons de feuilles Pesticides organochlorés ( $\mu \mathrm{g} / \mathrm{kg}$ de légumes) DDTs Endosulfan Aldrine Dieldrine Endrine Heptachlore Lindane

Moyenne

$\begin{array}{llllllll}\text { Sites } & \mathbf{0 . 2 2 4} & 0.356 & 0.115 & \mathbf{0 . 3 0 1} & \mathbf{0 . 1 1 2} & 0.070 & \mathbf{0 . 1 1 0}\end{array}$

*Normes

Codex

0.12

$0.1 \quad 0.1$

$0.02 \quad 0.05$

0.5 


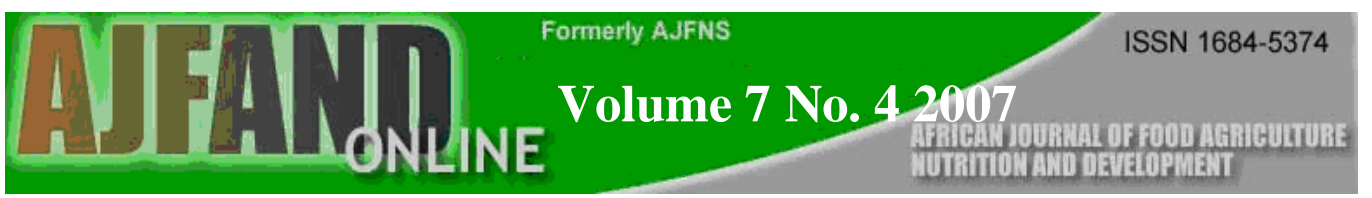

*Normes Codex Alimentarius : FAO, 2001

Tableau 7: Concentrations en pesticides organochlorés $(\mu \mathrm{g} / \mathrm{g})$ dans l'échantillon composite de sol

\begin{tabular}{|c|c|c|c|c|c|c|c|}
\hline \multirow{2}{*}{$\begin{array}{l}\text { Echantillo } \\
\mathrm{n}\end{array}$} & \multicolumn{7}{|c|}{ Pesticides organochlorés ( en $\mu \mathrm{g} / \mathrm{g}$ ) } \\
\hline & Aldrine & P,p'-DDT & $\begin{array}{l}\text { Endosulfa } \\
\mathrm{n}\end{array}$ & $\begin{array}{l}\text { Dieldrin } \\
\mathrm{e}\end{array}$ & Endrine & $\begin{array}{l}\text { Heptachlo } \\
\text { re }\end{array}$ & Lindane \\
\hline Sol & 0.496 & 4.800 & 7.975 & 2.150 & 3.568 & 0.725 & 2.457 \\
\hline
\end{tabular}




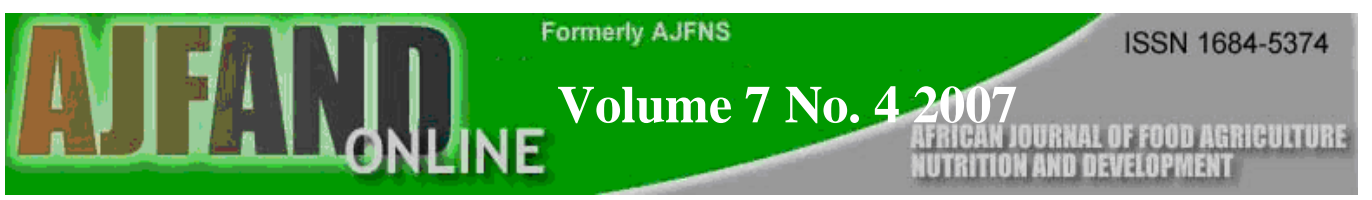

\section{REFERENCES BIBLIOGRAPHIQUES}

1. Lachance A Cultiver en ville. In: Ressources Explore Vol $21 \mathrm{~N}^{\circ} 3$. CRDI, Pays Bas, 1999.

2. FAO. Agriculture urbaine et périurbaine. Conférence virtuelle. FAO, Rome, 1999.

3. Moustier $\mathbf{P}$ et $\mathbf{J}$ Page Le périurbain en Afrique : une agriculture en marge ? In : Courrier de l'Environnement N³2. CIRAD, Montpellier, 1999.

4 Agossou G Ahouansou T Aly D et F Assogba-Komlan Etude sur la promotion de la filière des cultures maraîchères au Bénin. Rapport principal. MAEP, Bénin, 2001.

5 Colin et Heyd La situation des légumes feuilles dans la production maraîchère au Sud-Bénin. Tropicultura 1991; 9(3) : 129-133.

6 Hessou DJ Appui à l'intensification et à la production des cultures maraîchères et fruitières. Etude des stratégies à développer sur le plan nutritionnel. FAO/TCP/BEN/4553 (A), MDR, Bénin, 1995.

7 Hounkpodoté MR et C Tossou Profil des interactions entre la problématique foncière et le développement de l'agriculture urbaine dans la ville de Cotonou et environs. Rapport final, Chambre d'Agriculture du Bénin, RFAU/AOC IAGU, 2002.

8 Afouda L Sikirou $\mathbf{R}$ Assogba-Komlan $\mathbf{F}$ et $\mathbf{G}$ Gbéhounou Investigation sur les maladies et ravageurs des légumes feuilles, avec mention spéciale au gboma, au fotètè et au crin-crin. In : Actes de l'Atelier Scientifique 3, Programme Régional Sud-Centre du Bénin, $2002: 82-85$.

9 Brock B Actual and potential contribution of urban agriculture to environmental sanitation a case study in Cotonou. In: Agriculture Urbaine en Afrique de l'Ouest, IDRC publication, 1999: 126-137.

10 Assogba-Komlan $\mathbf{F}$ Etude diagnostique sur la valorisation des déchets agro-industriels pour la production de légumes dans les sols sableux du Littoral: Cas du site maraîcher de l'ONEPI. In : Actes de l'Atelier Scientifique 1, Programme Régional Sud-Centre du Bénin, 2000 : 144-151.

11 Assogba-Komlan F, Singbo A, Ahlé V et J Azagba (2001): Résultats préliminaires sur la valorisation de quelques déchets agroindustiels en maraîchage, Cas de la ville de Cotonou. In: Actes de 


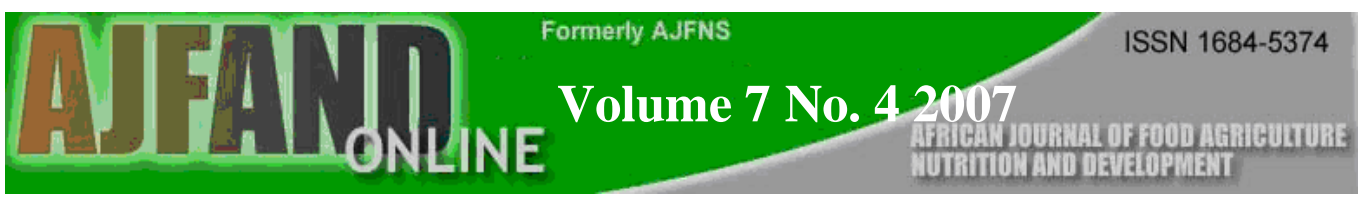

l'Atelier Scientifique 2, Programme Régional Sud-Centre du Bénin, 2001: 16-26.

12 Assogba-Komlan F, Ahlé V et J Azagba Apport raisonné des engrais organiques pour une bonne productivité des légumes feuilles en sols sableux marins. In: Actes de l'Atelier Scientifique 3, Programme Régional Sud-Centre du Bénin, 2002 : 150-157.

13 Amoussougbo Y Enquête sur l'usage des pesticides en cultures maraîchères dans le Sud-Bénin et expérimentation de trois nématicides dans la lutte contre les nématodes à galles. Thèse d'Ingénieur Agronome, Faculté des Sciences Agronomiques, UNBBénin, 1993.

14 PADAP. Etude de faisabilité: projet d'Appui au Maraîchage au sud du Bénin. Institut International pour l'Appui au Développement, Tome 2, Agrisud International, 2003.

15 Mbaye $\mathrm{A}$ et Renson Plan de promotion des cultures maraîchères et fruitières au Bénin. FAO/TCP/BEN/4553, 1997.

16 Soclo HH, Aguewe M, Adjahossou BC, Houngue TH et $\mathbf{A H}$ Azontondé Recherche de compost type et toxicité résiduelle au Bénin. TSM 1999; $9: 68-76$.

17 Mnzava NA Comparing nutritional values of exotic and indigenous vegetables. In : African Indigenous Vegetables, IPGRI and NRI; 1997: 70-75.

18 Dapoigny L, Fleury A et $\mathbf{P}$ Robin Relation entre la vitesse relative de croissance et la teneur en azote dans la laitue (Lactuva sativa L.). Effets du rayonnement et de la température. Agronomie 1997; 17: 3541.

19 Tittonell PA, De Grazia J et A Chiesa Nitrate and dry matter concentration in a leafy lettuce (Lactuca sativa L.) cultivar as affected by fertilization and plant population. Agricultura Tropica 2003; 36: 8287.

20 ABE/MEHU. Normes et standards environnementaux: principales sources de pollution de l'air et de l'eau au Bénin, Volume 1,1998.

21 Tremblay $\mathbf{N}$, Scharpf HC, Weier U, Laurence $\mathbf{H}$ et $\mathbf{J}$ Owen Régie de l'azote chez les maraîchers. Guide pour une fertilisation raisonnée. In : Agriculture et Agroalimentaire, Canada, 2001. 
22 Sanny SM Contribution à l'amélioration des rendements et de la qualité des cultures maraîchères (détection de biocontaminants et agents toxiques): Cas du périmètre maraîcher de Houéyiho à Cotonou. Mémoire pour l'obtention du Diplôme d'Ingénieur des Travaux. CPU/UAC, Bénin, 2002.

23 ABE. Etude des biocontaminants et migration des agents toxiques dans les cultures maraîchères au Bénin. Volet $\mathrm{N}^{0} 1$ : Périmètres maraîchers de Houéyiho et de Kouhounouu à Cotonou, 2003.

24 ACTA. Index phytosanitaire. 36 ${ }^{\text {ème }}$ édition, 2000.

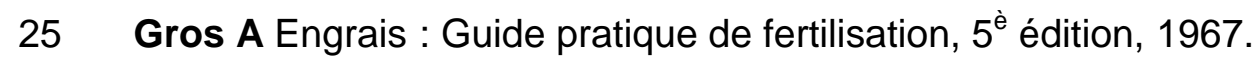

26 Amadji G Valorisation des ordures ménagères par la production du chou pommé sur sol sableux du littoral. In: Actes de l'Atelier "Agricultures et Développement Urbain en Afrique de l'Ouest et du Centre» sous presse. 2006

27 INRAB. Fiches techniques sur légumes feuilles traditionnels; sous presse 2006

28 FAO/OMS. Codex Alimentarius Vol 2B Réf. CAC/MRL 3 Rome, 2001. 